\title{
Dynamics of phase-locking random lasers
}

\author{
Marco Leonetti, ${ }^{1,2, *}$ Claudio Conti, ${ }^{3}$ and Cefe López ${ }^{4, \dagger}$ \\ ${ }^{1}$ Institute of Chemical and Physical Processes, Italian National Research Council, UOS Roma Kerberos, \\ Universitá La Sapienza, P. le A. Moro 2, I-00185 Rome, Italy \\ ${ }^{2}$ Center for Life Nano Science at Sapienza, Istituto Italiano di Tecnologia, Viale Regina Elena, 291-00161 Rome, Italy \\ ${ }^{3}$ Department of Physics, University Sapienza, P. le Aldo Moro 5, I-00185 Rome, Italy \\ ${ }^{4}$ Instituto de Ciencia de Materiales de Madrid, Consejo Superior de Investigaciones Científicas, \\ Calle Sor Juana Inés de la Cruz 3, 28049 Madrid, Spain
}

(Received 28 March 2013; published 18 October 2013)

\begin{abstract}
Laser modes may coalesce into a mode-locked state that enables femtosecond pulse compression. The nature of the interaction and the interaction time play fundamental roles in the onset of this collective state, but the investigation of the transition dynamics is technically challenging because phases are not always experimentally accessible. This is even more difficult for random lasers, a kind of disordered laser in which energies in play are much smaller than in the ordered macroscopic case. Here we investigate experimentally and numerically the dynamics of the phase-locking transition in a random laser. We developed an experimental setup able to pump individual modes with different pulse durations and found that the mode-locked regime builds only for quasicontinuous pumping, resulting in an emission linewidth dependent on the pump duration. Numerical simulation confirms experimental data.
\end{abstract}

DOI: 10.1103/PhysRevA.88.043834

PACS number(s): 42.55.Zz, 42.60.Fc, 64.70.fm

\section{INTRODUCTION}

The discovery of mode locking (ML) has been a milestone of laser physics allowing generation of femtosecond light pulses and opening the possibility of a huge range of applications. The mode-locked state may be achieved after a certain transient [1,2], by different approaches: in fibers just a small Kerr nonlinearity is needed to obtain mode locking [3], while in laser cavities nonlinear coupling may be obtained by active or passive modulation [4]. The ML is modeled by a nonlinear Schrodinger equation whose stable solutions are solitonlike. It is thus easy to establish a parallel between ML and BoseEinstein condensation of cold atoms [5], which is governed by the same equation. In fact, it has been recently demonstrated that in some cases mode locking is a form of condensation of classical waves [6-8] that, if treated in the framework of a thermodynamic approach, is a form of phase transition [9].

It has been recently demonstrated that collective states may be produced in systems where disorder is present, such as in random lasers (RLs). A RL $[10,11]$ is usually realized in a multiple scattering structure made of a closely packed agglomeration of high refractive index nanoparticles and in which gain is usually introduced by infiltrating the empty spaces by liquid dye solution. This system is intriguing both from the fundamental [11-17] and applied [18,19] points of view. At variance with standard lasers, in strongly scattering systems RL modes are random and thus it is difficult to know ex ante those above the laser threshold; on the other hand, while standard macroscopic cavity modes, because of the high cavity quality, are orthogonal and coupling has to be realized by an external device, in RL many microscopic and leaky cavities are instead naturally coupled.

\footnotetext{
"http://www.complexlight.org/; marco.leonetti@roma1.infn.it; http://www.mlphotonics.it/

†http://luxrerum.org
}

When the number of modes is relatively low, it is possible to follow experimentally the transition to a collective state, which has been associated with phase locking [20,21]. When the number of modes is huge, mean-field approximations are possible [22] and allow us to predict the existence of various phases including a mode-locked one [23]. It is possible to cast a Gross-Pitaevskii equation for the RL line shape [24] in analogy with classical wave condensation [8], as in the case of standard lasers.

Two fundamental issues make the study of phase locking in RLs more difficult than in standard lasers. The first is that in RLs there does not exist a single cavity but many small cavities randomly coupled, and thus it is impossible to follow the round trip behavior [3]. The second is that real time measurement of evolution of phases is beyond the current technological limit for RLs. In this paper we investigate the dynamics of the phase-locking process in RLs by an experimental approach that allows us to pump the system by two aligned lasers with different pulse durations (Fig. 1). We use a micron-sized assembly and a digital pump shaping (see [21]) to actively control the intermode interaction.

\section{EXPERIMENTAL SETUP}

We study the response of the RL system to quasiinstantaneous and quasicontinuous excitations. In the former (latter) case we use a pump laser with a pulse duration $\tau_{p}$ much shorter (much larger) than the mode lifetime, which is of the order of hundreds of picoseconds $[11,25]$. For the quasiinstantaneous (or "pico") pumping we used a $\tau_{p}=30 \mathrm{ps}$ laser (ND:YAG laser EKSPLA model PL2250, $20 \mathrm{~mJ}, 532 \mathrm{~nm}$ ). On the other hand, a $\tau_{p}=9 \mathrm{~ns}$ laser pulse is used for quasicontinuous (or "nano") pumping (ND:YAG laser Litron model Nano-T 250-10; $20 \mathrm{~mJ} 532 \mathrm{~nm}$ ) so that modes are continuously (that is, for a time much larger than their own lifetime) pumped and intermode interaction enables us to reach 


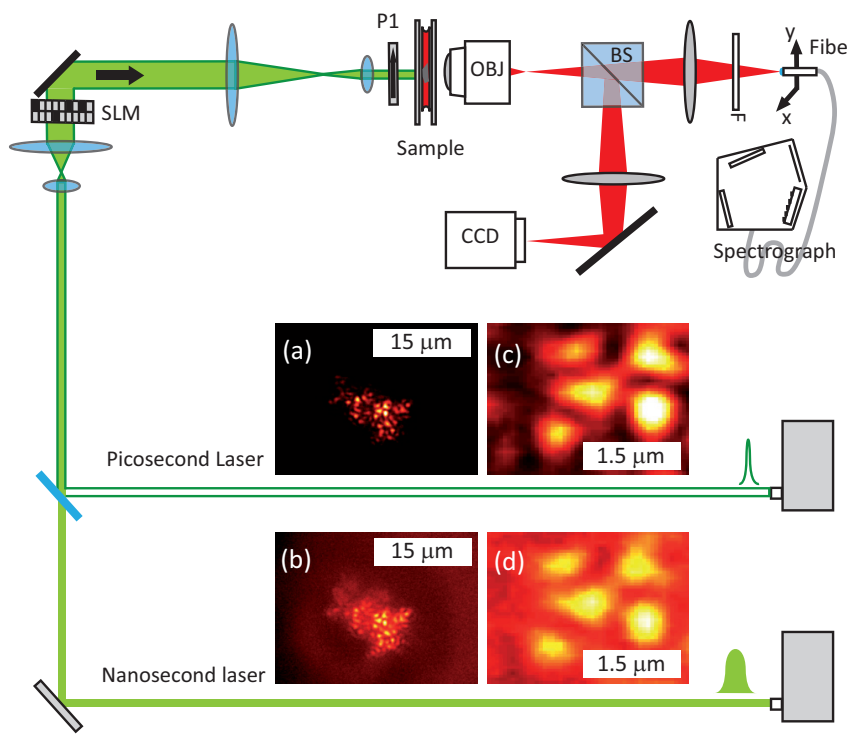

FIG. 1. (Color online) Experimental setup. The pulsed lasers $\left(532 \mathrm{~nm}\right.$, repetition rate $10 \mathrm{~Hz}$, and fluence $0.1 \mathrm{~nJ} / \mu \mathrm{m}^{2}$ for the nano and $0.03 \mathrm{~nJ} / \mu \mathrm{m}^{2}$ for the pico) are collected on the same beamline shaped by a SLM in amplitude configuration (exploiting polarizer P1). The two beams converge on a single titanium dioxide cluster (diameter between 5 and $12 \mu \mathrm{m}$ ). Insets: Images of the cluster collected in the pico configuration (a, c) and in the nanoconfiguration (b, d).

a steady state. We built an experimental setup (Fig. 1) in which the nanosecond laser (the nano configuration) and the picosecond laser (the pico configuration), differing in pulse duration by three orders of magnitude, are both able to excite the same modes of a single disordered structure.

The system under study is a single cluster of titanium dioxide particles self-assembled in a micron-scale structure of about $15 \mu \mathrm{m}$ average diameter [26] and a thickness of about $10 \mu \mathrm{m}$. Total transmission measurements allows to extrapolate a mean-free path of about $4 \mu \mathrm{m}$, which is compatible with a close packed arrangement of grains inside the cluster. Titanium particles have an average size of about $300 \mathrm{~nm}$ [titanium (IV) oxide, 89490 Sigma-Aldrich, particle size $<1 \mu \mathrm{m}]$ and the gain medium is a solution of ethanol and ethylene glycol doped with $1 \%$ (volume) of rhodamine B (Sigma-Aldrich 242425). The clusters are organized in a sparse arrangement on the bottom of the space defined by two microscopy coverslips separated by a plastic film [21], and it is well known that they are able to support modes with various degrees of interaction [26,27]. Moreover, this configuration allows for an easy manipulation of the number of activated modes (see below). Figures 1(a)-1(d) show images captured by the CCD when the cluster is shone on with the pico $(\mathrm{a}, \mathrm{c})$ and nano $(\mathrm{b}, \mathrm{d})$ pumping. In the lower panels (c for pico and $\mathrm{d}$ for nano) an enlarged region of the more intense spots ("hotspots") is shown. The alignment is designed to produce virtually identical position and shape of the hotspots.

Another feature of the setup is that the pump spot may be spatially shaped through the use of a spatial light modulator. This approach, which has been well described in [20,21,27], allows to control, through the $\Theta$ parameter, i.e., the angular aperture of the inverted area (see, for example, Fig. 2 of [20]),
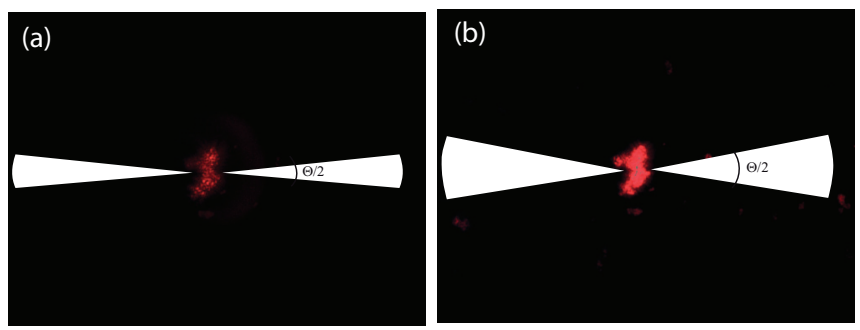

FIG. 2. (Color online) Cluster and pumped area (evidenced in white) for small theta (a) and for large theta (b).

the set of directions from which stimulated emission from the free dye pumps the cluster. In this configuration the pump selects only the modes that are well coupled with the input direction [28,29]. In Figs. 2(a) and 2(b) an image of the cluster pumped with small (large) $\Theta$ is reported. The amplified spontaneous emission generated in the pumped area serves as directional pumping and activates only modes coupled to the preselected directions. Thus, a small $\Theta$ results in a small set of modes oscillating individually [like the spectrum in Fig. 3(a)]; in contrast, for a large $\Theta$ many modes are activated simultaneously and the interaction generates a phase-locked emission with a smooth and line narrowed spectra, as in Fig. 4(a).

Moreover, the detection has been designed to select only light emitted from the cluster and to eliminate contribution
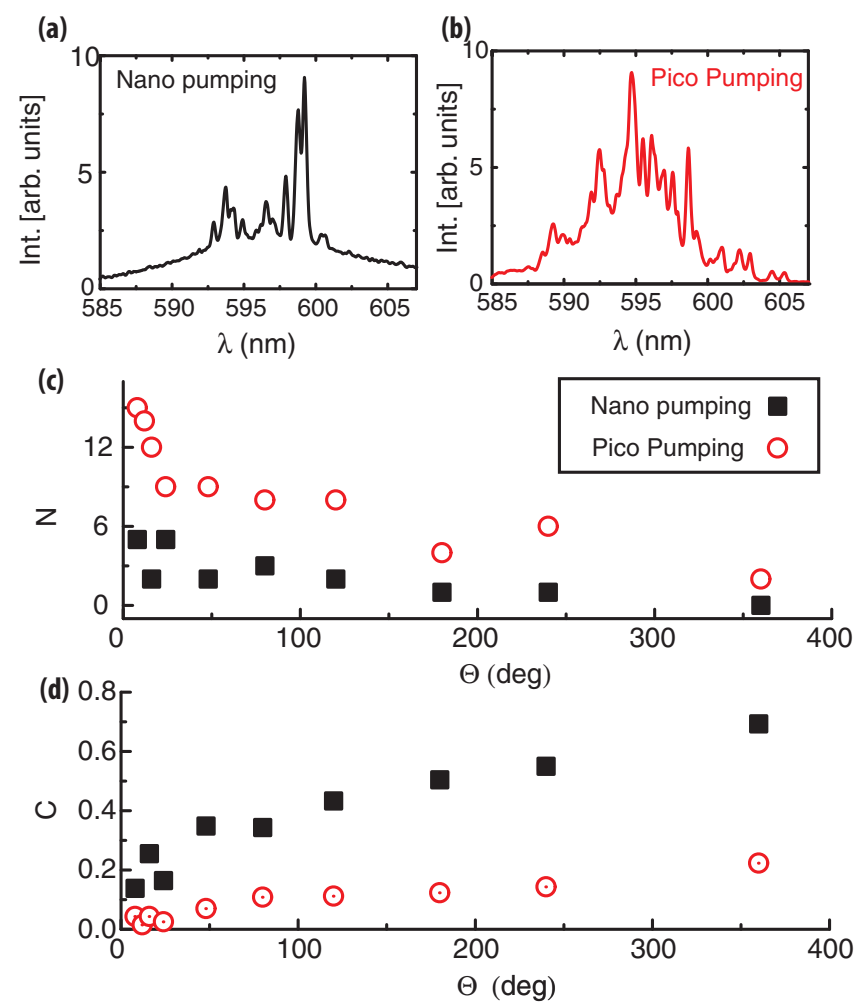

FIG. 3. (Color online) (a) Spectra for a nano pumping (150 shots average) with $\Theta=8^{\circ}$ and pump fluence $0.1 \mathrm{~nJ} / \mu \mathrm{m}^{2}$. The pico pumping is as in panel (a), and $0.03 \mathrm{~nJ} / \mu \mathrm{m}^{2}$ is reported in panel (b). (c, d) The number of peaks, respectively, in the spectrum and the intermode correlation as a function of $\Theta$. 
(a)
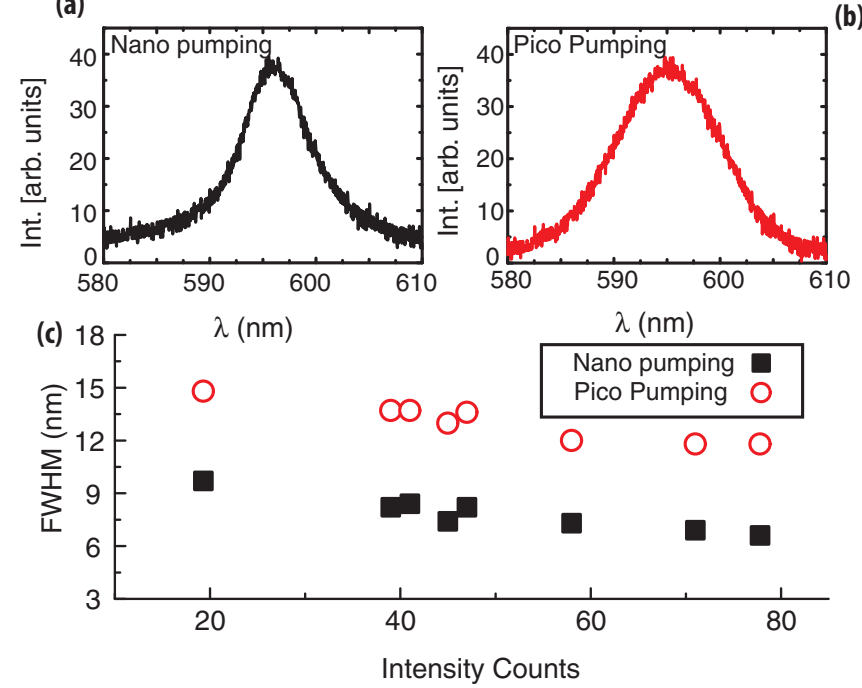

FIG. 4. (Color online) (a, b): the spectrum obtained, respectively, for the nano and for the pico configurations. (c) The FWHM of the spectra for different clusters both in the nano (squares) and the pico (circles) pumping.

from the scattering-free areas. A microscope objective captures light and images it magnified (in the present experiment 50×) on a plane in which a $200-\mu \mathrm{m}$ core fiber collects the light, thus providing for a spatial resolution of $4 \mu \mathrm{m}$. In the experiments below we placed the fiber in such a way to collect light from the center of the considered cluster structure.

\section{RESULTS AND DISCUSSION}

We first report the lasing spectrum obtained from cluster $\mathrm{C} 1$ [Figs. 1(a) and 1(b)] with $\Theta=8^{\circ}$ in the nano [Fig. 3(a)] and the pico [Fig. 3(b)] configurations. The pump energy has been adjusted to yield a comparable number of counts in the two configurations. The spectrum in Fig. 3(a) in the nano pumping is characterized by a lower number of peaks with respect to the same in Fig. 3(b) with pico pumping. Moreover, the effective spectral window in which lasing peaks are found is smaller in the nano configuration (lasing peaks appear from 592.5 to $601.5 \mathrm{~nm}$ spanning a 9-nm range) if compared with the pico configuration (from 587.5 to $605 \mathrm{~nm}, 17.5-\mathrm{nm}$ band).

This difference in the number of lasing modes resulted to be independent of the pump energy and remains also when the $\Theta$ parameter, which controls the total number of modes, is varied. In Fig. 3(c) the number of activated modes $N$ (counted as the maxima of the spectrum over $15 \%$ with respect to their neighbor points) in cluster $\mathrm{C} 1$ is plotted versus $\Theta$ for the nano and pico configurations. For any $\Theta$ we find $N_{\text {pico }}>N_{\text {nano }}$, this is an unexpected finding, because for the probed cavities the scattering matrix is exactly the same. Thus, the difference has to lie in the interaction strength that may be estimated from the correlation $C$ between any two modes $m, n$ at frequencies $\lambda_{m}$, and $\lambda_{n}$ is calculated by using the Pearson correlation (see also $[20,21])$ :

$$
C_{p}^{m, n}=\frac{\sum_{i=1}^{N}\left[I\left(\lambda_{m}\right)_{i}-\bar{I}\left(\lambda_{m}\right)\right]\left[I\left(\lambda_{n}\right)_{i}-\bar{I}\left(\lambda_{n}\right)\right]}{\sqrt{\sum_{i=1}^{N}\left[I\left(\lambda_{m}\right)_{i}-\bar{I}\left(\lambda_{m}\right)\right]^{2}} \sqrt{\sum_{i=1}^{N}\left[I\left(\lambda_{n}\right)_{i}-\bar{I}\left(\lambda_{n}\right)\right]^{2}}},
$$

where $\bar{I}$ represents an average over one hundred shots. If two modes have $C_{p}^{m, n}$ close to zero, their fluctuations are uncorrelated while correlated modes result in $C_{p}^{m, n} \cong 1$. The correlation $C_{p}$ reported in Fig. 3(d) is the average over all pairs from the ten most intense modes.

For the nano pumping (as in [20]) an increased mode density results in greater mode spatial overlap, thus favoring interaction and enhancing spectral correlation. In the pico case only a limited increase of $C_{p}$ is found and the values of correlations are much lower. This is clear evidence that interaction takes time to build up so that lower interaction time results in a lower interaction strength.

We observe that lowering $\Theta$ has an effect very similar to switching from the nano to the pico pumping. In this respect the pumping angle $\Theta$ plays a role similar to the pulse duration, both enhancing the interaction: the number of lasing modes decreases by increasing either the spatial (determined by $\Theta$ ) or temporal (determined by $\tau_{p}$ ) interaction. This may be explained by the presence of competition: when the interaction is strong, only cooperative modes survive, while in the low interacting regime less efficient modes may successfully emit without being inhibited by stronger (more efficient) neighbors.

A direct consequence of the varying degree of interaction is on the "condensation strength." Recent work $[24,30]$ demonstrates that the smooth spectrum may be explained as a condensationlike process between the many modes activated by the pump. This approach allows us to cast a Gross-Pitaevskii equation which predicts the line narrowing (lasing frequencies are attracted in a small spectral region) of the RL peak found when increasing pump energy. In the many-modes (large $\Theta$ ) configuration, the RL emission is equivalent to a condensed collective spectrum. The condensation strength may be measured by the full width at half maximum (FWHM) of the random lasing peak. A narrower line represents a more efficient process. To investigate the pico and the nano configurations it is useful to compare emission spectra with the same maximum intensity; in fact, being input and output coupling fixed, the number of counts is a good estimate of the energy introduced in the cluster.

Figure 4 shows spectra taken from cluster $\mathrm{C} 2$ with $\Theta=240^{\circ}$ for nano (a) and pico (b) pumping. The pulse energy in the pico configuration has been adjusted to yield an emission with the same counts (within $\pm 10 \%$ tolerance) of the nano configurations. The important feature is that the bandwidth of the pico RL is much larger than the nano RL. Reducing pumping duration weakens the strength of the condensation process. We confirmed that this result is independent of the disorder realization by repeating the measurement on many different clusters. In Fig. 4(c) we report the FWHM as a function of the output counts for many clusters, demonstrating that the lasing bandwidth appears to be always higher in the pico configuration, regardless of the pumping intensity. 

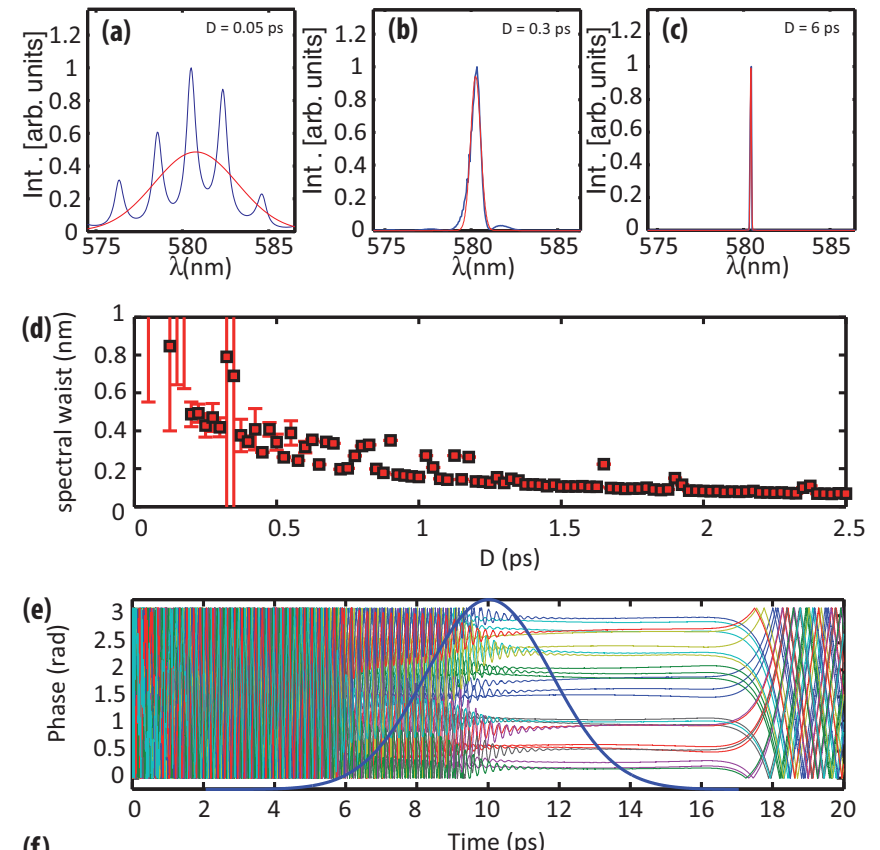

(f)

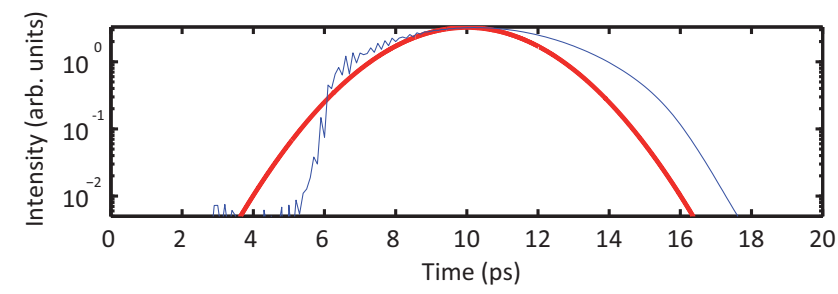

FIG. 5. (Color online) Results of the numerical simulations. (a-c) The spectra obtained for various pumping durations. (d) The spectral waist retrieved by Gaussian fitting (red line in the first three panels) as a function of the pumping duration $D$. (e, f) The phases and the total intensity, respectively, as a function of time. The pump pulse shape ( $D=2.5 \mathrm{ps}$ ) is represented as a thick line.

\section{NUMERICAL SIMULATIONS}

We support our experiments by coupled-mode theory simulations that allow us to follow the temporal behavior of the amplitude $a_{i}$ of the mode $i$. The approach is well known [4] and has been described in particular in [4,20]. We took into consideration 25 modes with average lifetime $\alpha=0.5 \mathrm{ps}$ and frequency $\omega_{i}$. The mode wavelengths are random but with an average spacing of $0.5 \mathrm{~nm}$. Gain $G(t)$ is time dependent: it has a Gaussian shape simulating pump laser pulses of duration $D$. To simulate the "constant energy" condition the gain strength is chosen inversely proportional to the pumping duration. The equation to be solved is the following:

$$
\frac{d a_{i}(t)}{d t}=i \omega_{i} a_{i}(t)-\alpha_{i} a_{i}(t)+\sum_{\text {neighbors }} C_{i j} a_{j}(t)+\frac{G(t) a_{i}(t)}{1+\gamma\left|a_{i}\right|^{2}}
$$

Each mode is coupled with eight nearest neighbors, with coupling constants $C_{i j}$, while $\gamma$ is gain saturation. Here we report the result of a set of simulations with variable pump duration: in Figs. 5(a)-5(c) we report the obtained spectra. For very short pumping ( $D=0.1 \mathrm{ps}$ ) the system does not exhibit any phase locking and multiple peaks appear in the spectrum.
The "condensed" state is steadily retrieved for $D \geqslant 0.3 \mathrm{ps}$, and linewidth narrows by increasing pump duration. In Fig. 5(d), we show the spectral width (obtained by crude Gaussian fitting) as a function of the input pumping duration. A decreasing trend is evident. A qualitative explanation for this phenomenon is that the condensed state requires a certain time to build up because the modes need a certain interaction time. We kept fixed the temporal duration of the simulation for all values of $D$; trials with larger simulation times confirmed the behavior reported in Fig. 5(d), excluding finite-size effects.

The onset of a condensed state is further confirmed by the phase locking appearing only for pulses with sufficient duration [Fig. 5(e)], after an intermediate regime in which modes stabilize their relative phase difference. While mode locking in the stationary condition (i.e., for lasers pumped by continuous beams) corresponds to a widening of the spectrum, the transient case shows an opposite behavior. Here we stress that in the random case phase synchronization does not necessarily correspond to a pulse compression. On the other hand, the fact that we achieve such a small final linewidth in simulations is connected to the limited number of modes simulated (which is several orders of magnitude smaller than the number of modes involved in real experiments) and also to the limited duration of the simulations. Figure 5(f) shows the total intensity stored in the whole random laser (thin line) as a function of time. The thick line represent the pump pulse.

The time length needed to achieve the mode locking results from the interplay between coupling and losses, and thus if losses are dominant the coupling dynamics are inhibited and the mode-locked state may not be achieved. On the other hand, a prolonged pump duration increases the effective lifetime of the modes. Thus, the relevant parameter is the mode lifetime: if the pump is much shorter, phase locking is inhibited, while a longer duration of the feeding allows us to retrieve the collective state. The simulations (which are performed with a mode lifetime of $\alpha=0.5 \mathrm{ps}$ ) confirm this picture.

The fact that the condensation is hampered by shorter pump pulses may be at the origin of the presence of spikes in this temporal regime [31]: all modes emitting in an uncorrelated manner are more susceptible to stochastic features like "lucky photons," which are indeed almost exclusively found in the picosecond pumping regime.

\section{CONCLUSIONS}

We demonstrated that the condensation of classical waves in a dissipative random system is a dynamic process. The duration of pumping critically affects the RL emission spectra, reducing the effective intermode interaction and the number of lasing modes over the threshold. Also, the spectral width is affected: the emission obtained in the picosecond configuration is always broader than that retrieved by nanosecond configuration, meaning that a stronger condensation needs a longer time interval to build up. This effect may be exploited for a generation of devices, allowing spectral tunability through temporal shaping in a RL. Everything suggests that this phenomenon may not be limited to laser systems but may be found in other kinds of condensates of classical and quantum waves. 


\section{ACKNOWLEDGMENTS}

The research leading to these results has received funding from the European Research Council under the European Commission's Seventh Framework Program (FP7/2007-2013)
Grant No. 201766, EU FP7, and EU FP7 NoE Nanophotonics4Energy Grant No. 248855; the Spanish Ministerio de Ciencia e Innovación CSD2007-0046 (Nanolight.es); MAT201231659 (SAMAP); and Comunidad de Madrid S2009/MAT1756 (PHAMA).
[1] D. Marcuse, IEEE J. Quantum Electron. 20, 1139 (1984).

[2] O. Buccafusca, J. Chilla, J. Rocca, S. Feld, C. Wilmsen, V. Morozov, and R. Leibenguth, Appl. Phys. Lett. 68, 590 (1996).

[3] K. M. Spaulding, D. H. Yong, A. D. Kim, and J. N. Kutz, J. Opt. Soc. Am. B 19, 1045 (2002).

[4] H. A. Haus, IEEE J. Sel. Top. Quantum Electron. 6, 1173 (2000).

[5] F. Dalfovo, S. Giorgini, L. P. Pitaevskii, and S. Stringari, Rev. Mod. Phys. 71, 463 (1999).

[6] R. Weill, B. Levit, A. Bekker, O. Gat, and B. Fischer, Opt. Express 18, 16520 (2010).

[7] R. Weill, B. Fischer, and O. Gat, Phys. Rev. Lett. 104, 173901 (2010).

[8] C. Sun, S. Jia, C. Barsi, S. Rica, A. Picozzi, and J. Fleischer, Nat. Phys. 8, 471 (2012).

[9] A. Rosen, R. Weill, B. Levit, V. Smulakovsky, A. Bekker, and B. Fischer, Phys. Rev. Lett. 105, 013905 (2010).

[10] D. S. Wiersma, Nat. Phys. 4, 359 (2008).

[11] C. M. Soukoulis, X. Jiang, J. Y. Xu, and H. Cao, Phys. Rev. B 65, 041103 (2002).

[12] N. M. Lawandy, R. Balachandran, A. Gomes, and E. Sauvain, Nature (London) 368, 436 (1994).

[13] C. Vanneste, P. Sebbah, and H. Cao, Phys. Rev. Lett. 98, 143902 (2007).

[14] H. Cao, X. Jiang, Y. Ling, J. Y. Xu, and C. M. Soukoulis, Phys. Rev. B 67, 161101 (2003).

[15] R. G. S. El-Dardiry, A. P. Mosk, O. L. Muskens, and A. Lagendijk, Phys. Rev. A 81, 043830 (2010).
[16] J. Fallert, R. J. B. Dietz, J. Sartor, D. Schneider, C. Klingshirn, and H. Kalt, Nat. Photon. 3, 279 (2009).

[17] S. Mujumdar, V. Türck, R. Torre, and D. S. Wiersma, Phys. Rev. A 76, 033807 (2007)

[18] B. Redding, M. A. Choma, and H. Cao, Nat. Photonics 6, 496 (2012).

[19] L. Cerdán, E. Enciso, V. Martín, J. Bañuelos, I. López-Arbeloa, A. Costela, and I. García-Moreno, Nat. Photonics 6, 621 (2012).

[20] M. Leonetti, C. Conti, and C. Lopez, Nat. Photonics 5, 615 (2011).

[21] M. Leonetti, C. Conti, and C. López, Phys. Rev. A 85, 043841 (2012).

[22] C. Conti and L. Leuzzi, Phys. Rev. B 83, 134204 (2011).

[23] L. Leuzzi, C. Conti, V. Folli, L. Angelani, and G. Ruocco, Phys. Rev. Lett. 102, 083901 (2009).

[24] C. Conti, M. Leonetti, A. Fratalocchi, L. Angelani, and G. Ruocco, Phys. Rev. Lett. 101, 143901 (2008).

[25] M. Siddique, R. R. Alfano, G. A. Berger, M. Kempe, and A. Z. Genack, Opt. Lett. 21, 450 (1996).

[26] M. Leonetti and C. Lopez, Appl. Phys. Lett. 101, 251120 (2012).

[27] M. Leonetti, C. Conti, and C. Lopez, Appl. Phys. Lett. 101, 051104 (2012).

[28] M. Leonetti and C. López, Appl. Phys. Lett. 102, 071105 (2013).

[29] M. Leonetti, C. Conti, and C. Lopez, Nature Communications 4, 1740 (2013).

[30] M. Leonetti and C. Conti, J. Opt. Soc. Am. B 27, 1446 (2010).

[31] S. Mujumdar, M. Ricci, R. Torre, and D. S. Wiersma, Phys. Rev. Lett. 93, 053903 (2004). 